(C) 2022, The Authors. Published by Elsevier Inc. and Fass Inc. on behalf of the American Dairy Science Association ${ }^{\circledR}$. This is an open access article under the CC BY license (http://creativecommons.org/licenses/by/4.0/).

\title{
Assessment of the microbiological origin of blowing defects in Grana Padano Protected Designation of Origin cheese
}

\author{
Vinícius da Silva Duarte, ${ }^{*}$ • Angiolella Lombardi, Viviana Corich, $\dagger$ and Alessio Giacomini $\odot$ \\ Department of Agronomy Food Natural Resources Animals and Environment, University of Padova, 35020 Legnaro (Padua), Italy
}

\begin{abstract}
Recognized worldwide for its history, flavor, and high nutritional quality, Grana Padano (GP) is one of the most traditional Italian raw-milk, hard-cooked, longripened cheese. Throughout GP manufacturing, some well-known and undesired bacterial species, such as clostridia, can proliferate and lead to spoilage defects that mischaracterize the final product; however, little is known about the development of late-blowing defects in hard cheese samples without clostridia. Therefore, in this study we aimed to use metataxonomic analysis to identify bacterial taxa associated with the development of late-blowing defect in GP samples. Furthermore, the presence of several heterofermentative lactobacilli species in defective zones were verified by primer-specific PCR assay. Considering $\alpha$ - and $\beta$-diversity analyses, no statistically significant differences were detected between cheese samples with and without blowing defect. Following taxonomic assignment, Lactobacillus and Streptococcus were the dominant genera, whereas clostridia-related taxa were not detected in any of the 20 analyzed samples. Using EdgeR, the genera Propionibacterium and Acinetobacter were found to be prevalently more abundant in samples categorized as having "big regular holes." In samples with "small regular holes," multiplex PCR amplification revealed differences in terms of Lactobacillus population composition, mainly obligate homofermentative lactobacilli, between defective and non-defective zones of the same cheese wheel. This study demonstrated that GP samples with blowing defects not caused by clostridial development share similar biodiversity indices with GP collected from control zones, but an imbalance of obligate homofermentative lactobacilli was noticed between samples,
\end{abstract}

Received August 2, 2021.

Accepted October 20, 2021.

*Current address: Faculty of Chemistry, Biotechnology, and Food Science, Norwegian University of Life Sciences, PO Box 5003, N-1432, As, Norway.

$\dagger$ Corresponding author: viviana.corich@unipd.it which requires further analysis to better comprehend the exact mechanism involved in this process.

Key words: hard cheese, $16 \mathrm{~S}$ rRNA, lactobacilli, Grana Padano

\section{INTRODUCTION}

Grana Padano (GP) is a well-known traditional Italian raw-milk, hard-cooked, long-ripened cheese, whose technological and microbiological characteristics have been reviewed previously (Neviani et al., 2013). It has been a Protected Designation of Origin (PDO) cheese since 1996, and it is produced in 5 regions located in northern Italy; namely, Emilia-Romagna, Lombardia, Piemonte, Trentino-Alto Adige, and Veneto). According to Bava et al. (2018), it is estimated that nearly $24 \%$ of the total cow milk produced in Italy is destined for GP production. In 2020 alone, 5,255,451 wheels of this cheese were produced, and $39 \%$ were exported outside Italy, making this cheese the food product most sold worldwide (Grana Padano Protection Consortium, www.granapadano.it). The ripening period for this cheese can vary from 9 mo to more than 2 yr (Gatti et al., 2014). During this period, bacteria from the raw milk and from the added whey starter cultures develop inside the cheese and give it its peculiar characteristics (Rossetti et al., 2008). At the same time, deleterious microbes possibly present in the raw milk or in the cheesemaking plant can also develop and determine the creation of defects (Bassi et al., 2015). Although the presence of small holes does not constitute a threat to food security, this type of defect must be barely visible (less than $5 \mathrm{~mm}$ in diameter) and not lead to undesirable effects on textural and sensory parameters, with consequent loss of product value.

Late-blowing (LB) defect is a well-known and quite widespread problem in long-ripened cheeses such as GP, primarily determined by the proliferation of bacteria, mainly belonging to the genus Clostridium, during cheese ripening, and it can be responsible for relevant commercial losses (Bermúdez et al., 2016; D'Incecco et al., 2018). These bacteria typically cause holes (also called "eyes") of different diameter inside the cheese 
wheels that, in serious cases, can also visibly deform the wheel outside. In addition to these well-known bacteria, mainly belonging to the species Clostridium butyricum and Clostridium tyrobutyricum, other microorganisms such as propionic acid bacteria can produce similar defects.

Over the last few years, thanks to reductions in the costs of next-generation sequencing, several studies have focused on assessment and comprehension of the microbial community by cultivation-independent techniques of raw materials (e.g., raw milk and natural whey starter cultures) used in GP manufacturing, as well as during the GP ripening process (Neviani et al., 2013; De Filippis et al., 2014; Gatti et al., 2014; Alessandria et al., 2016; Mayo et al., 2021). However, few studies have reported the bacterial community structure of GP samples with or without LB defect (Bassi et al., 2015; Zago et al., 2021). Overall, studies demonstrating the involvement of bacterial genera different from Clostridium (e.g., propionibacteria or heterofermentative lactic acid bacteria) as causative agents of late blowing are still scarce and mostly based on DNA identification. Therefore, in this study we aimed to characterize and identify, through metataxonomic analysis, the major bacterial taxa associated with the development of LB defects in GP samples. Finally, the associations of several heterofermentative lactobacilli with defective zones testing negative for the presence of clostridia cells were verified by primer-specific PCR assay.

\section{MATERIALS AND METHODS}

\section{Cheese Sampling}

Nine wheels of GP PDO cheese from a cheese factory located in the Veneto region, having 8 to 9 mo of maturation, that were considered defective, were sampled. Ten to twenty grams of cheese was scraped with a sterile spatula, taking care to collect only the part surrounding the holes, and samples were named S1 to S9. For wheels with different hole dimensions, 2 separate samples were taken and named, for example, S2 and S2A (Figure 1). For each wheel, a portion not presenting defects was also separately sampled and used as control, named CTR1 through CTR9. A total of 20 samples were collected (Table 1), immediately transferred into sterile 50-mL Falcon tubes, maintained refrigerated during transportation, and then stored at $-20^{\circ} \mathrm{C}$ until use.

\section{Total DNA Extraction from Cheese}

In this study, the PowerSoil DNA Isolation Kit (Qiagen) protocol modified for $1 \mathrm{~g}$ of cheese was adopted.
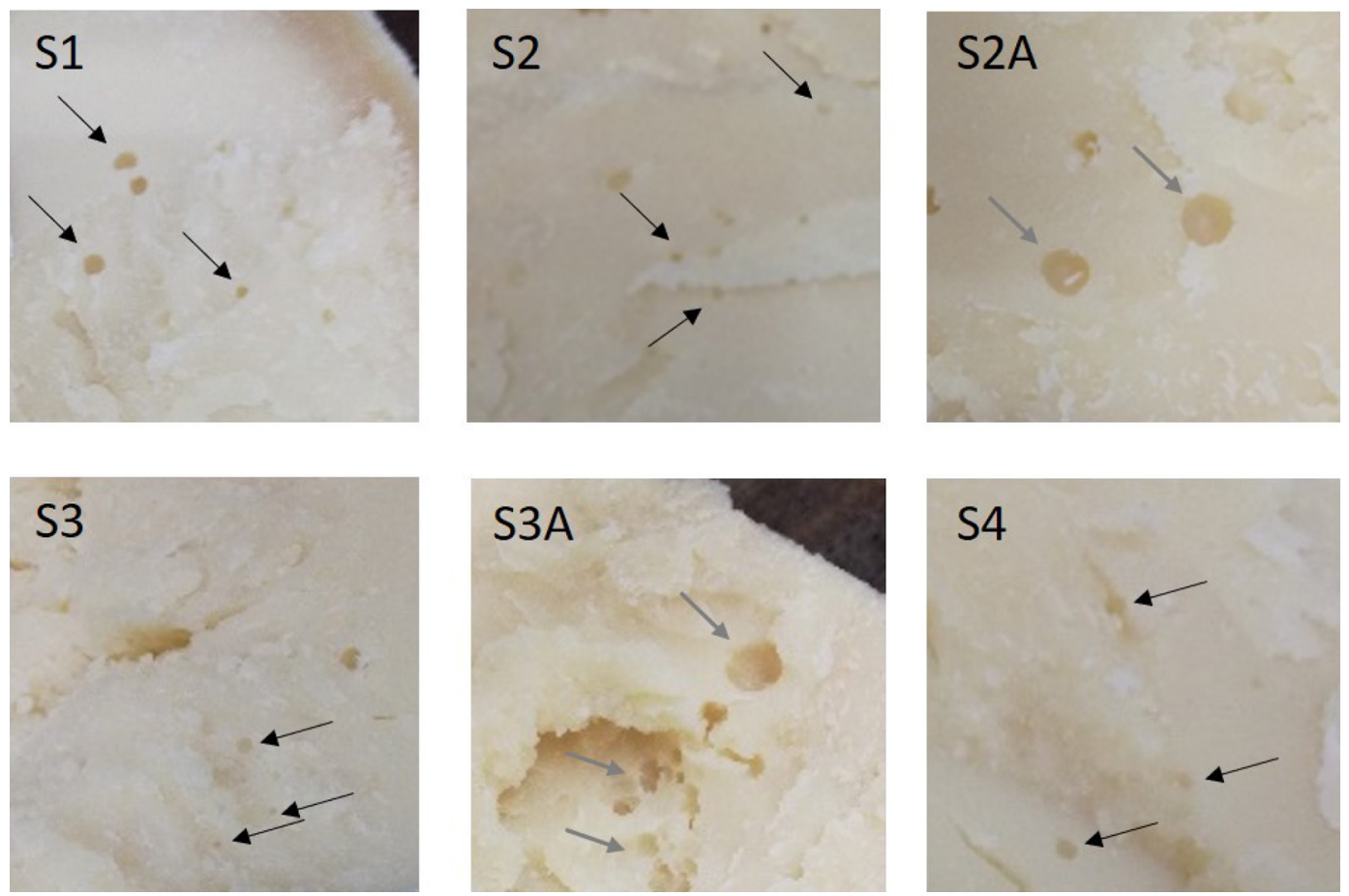

Figure 1. Representative images of the sampled zones. Black arrows indicate small sparse eyes, whereas gray arrows indicate large sparse eyes. S1 to S4 represent samples with small holes; S2A and S3A are samples with large holes. 
In brief, $2 \mathrm{~mL}$ of phenol:chloroform:isoamyl alcohol $(25: 24: 1, \mathrm{vol} / \mathrm{vol})$ at $\mathrm{pH} 8.0$ and $2 \mathrm{~mL}$ of $\mathrm{PBS}(\mathrm{NaCl}$ $0.13 M, \mathrm{KCl} 2 \mathrm{~m} M, \mathrm{Na}_{2} \mathrm{HPO}_{4} 9 \mathrm{~m} M, \mathrm{KH}_{2} \mathrm{PO}_{4} 1 \mathrm{~m} M, \mathrm{pH}$ 7.4) were added to a polystyrene $15-\mathrm{mL}$ tube containing $1 \mathrm{~g}$ of cheese sample. Sequentially, $60 \mu \mathrm{L}$ of solution C1, as well as the entire contents of one PowerBead tube (Qiagen; $750 \mu \mathrm{L}$ of solution and beads), were added to the sample tube and the mixture was vortexed at maximum speed for $15 \mathrm{~min}$. After centrifugation at 10,000 $\times g$ for 5 min at $4^{\circ} \mathrm{C}$, sequential phenol:chloroform (1:1) and chloroform (1:1) steps were carried out at 10,000 $\times g$ for $5 \mathrm{~min}$ at $4^{\circ} \mathrm{C}$. The obtained supernatant was divided into aliquots of $500 \mu \mathrm{L}$ and transferred to $2-\mathrm{mL}$ microfuge tubes previously filled with 250 of solution C2. From this stage, all successive steps followed manufacturer recommendations. The quality and quantity of the extracted DNA were assessed by a Spark $10 \mathrm{M}$ spectrophotometer (Tecan Trading AG). The DNA samples were stored at $-20^{\circ} \mathrm{C}$ until further analysis.

\section{S rRNA Gene Amplicon Sequencing and Molecular Identification of Dairy Heterofermentative Species}

Total metagenomic DNA samples were sent to Eurofins (Eurofins Genomics Germany GmbH, Ebersberg, Germany), where amplicon preparation and sequencing were performed. After amplification of the V3 to V5 region of the $16 \mathrm{~S}$ rRNA genes using $357 \mathrm{f}$ and $962 \mathrm{r}$ primers, amplicon libraries were generated using the Nextera XT DNA Library Preparation Kit (Illumina Inc.) and sequenced using the Illumina MiSeq desktop sequencer producing 300-bp paired-end reads.

Pairs of primers specific for different heterofermentative Lactobacillus spp. and Leuconostoc spp. were obtained from different studies (Table 2) to evaluate the presence and possible involvement of these micro-

Table 1. Samples of cheeses used in this study

\begin{tabular}{lll}
\hline Sample & Production $^{\text {date }}$ & Eye dimension \\
& \\
\hline S1 & $05-09-2017(88)$ & Small \\
S2 & $05-09-2017(101)$ & Small \\
S2A & $05-09-2017(101)$ & Large \\
S3 & $05-09-2017(28)$ & Small \\
S3A & $05-09-2017(28)$ & Large \\
S4 & $18-09-2017(106)$ & Small \\
S5 & $18-09-2017(100)$ & Small \\
S6 & $14-09-2017(63)$ & Small \\
S7 & $30-09-2017(114)$ & Small \\
S8 & $28-09-2017(40)$ & Small \\
S8A & $28-09-2017(40)$ & Large \\
S9 & $30-09-2017(114)$ & Small \\
\hline
\end{tabular}

${ }^{1}$ Dates are presented in day-month-year format. The number of the vat used to produce each specific cheese wheel is reported in parentheses.

${ }^{2}$ Eyes with a diameter greater than $\sim 5 \mathrm{~mm}$ were considered large. organisms in the development of blowing defects in the GP samples. Primers were obtained from Invitrogen (Thermo Fisher Scientific). Amplifications were carried out in a total volume of $25 \mu \mathrm{L}$ using $50 \mathrm{ng}$ of metagenomic DNA as the template and using the same primer concentration and Taq DNA polymerase units (GoTaq, Promega) adopted in the study in which each primer was described. We conducted PCR amplification using a BioRad iCycler iQ PCR thermal cycler (BioRad), setting up the thermocycling program specific for each pair of primers. The PCR products were subjected to electrophoresis on $1.5 \%$ (wt/vol) agarose gels in Trisborate-EDTA (TBE) buffer solution at $70 \mathrm{~V}$ for $1.5 \mathrm{~h}$, followed by EuroSafe staining (EuroClone).

\section{Bioinformatics Analyses}

Raw 16S rRNA sequences were analyzed with the CLC Genomics Workbench software (version 8.0.2, Qiagen Bioinformatics) using the microbial genomics module plugin as described by Treu et al. (2018). In brief, quality filtering, operational taxonomic unit (OTU) clustering, and taxonomical assignment (Greengenes database, version 13-5; https://greengenes .secondgenome.com) were conducted with default parameters. To improve the taxonomical assignment and make inferences at the species level when appropriate, OTU consensus sequences were manually verified using the MegaBLAST database (https://blast.ncbi.nlm.nih .gov/Blast.cgi??DATABASE $=$ nr\&PAGE=MegaBlast; $16 \mathrm{~S}$ ribosomal RNA sequences). Throughout the manuscript, the new nomenclature for Lactobacillus species proposed by Zheng et al. (2020) has been adopted. Raw reads were deposited into the Sequence Read Archive database (http://www.ncbi.nlm.nih.gov/sra) under the BioProject PRJNA748599.

\section{Statistical Analysis}

All the statistical analyses of the microbiota data were carried out with MicrobiomeAnalyst (Dhariwal et al., 2017). Following manual data inspection, low-count (minimum count: 2; prevalence in samples: 10\%) and low-variant filters (interquantile range: 10\%) were applied and centered log-ratio transformed. Differential abundance analysis was conducted with edgeR (Robinson et al., 2010), taking into account only those taxa with $\log 2$ fold change $(\log \mathbf{2 F C})$ greater than 2.0 and with a false discovery rate-adjusted $P$-value $<0.05$. For $\alpha$-diversity analysis, the non-parametric MannWhitney/Kruskal-Wallis tests were chosen, whereas microbiota similarities among the groups $(\beta$-diversity analysis) were assessed using the Unweighted and 
Weighted UniFrac metrics. Permutational multivariate ANOVA (PERMANOVA) with 999 permutations was used to test whether distances between samples within a certain group were more similar to each other or not. Differences were considered statistically significant for a $P$-value $<0.05$.

\section{RESULTS AND DISCUSSION}

In this study, we examined 9 GP PDO wheels that, after about 9 mo of maturation, revealed anomalous sounds when knocked with a specific small hammer, a standard procedure used to detect anomalous wheels, indicating the possible presence of internal cavities, and were therefore considered defective. The wheels were opened and sampled both in a clearly defective portion as well as in a normal and adjacent zone (control sample). After high-throughput sequencing of the V3 to $\mathrm{V} 5$ region of the $16 \mathrm{~S}$ rRNA genes aiming at detection of the bacterial diversity in GP samples with or without LB defects, a total of 1,123,291 high-quality sequences were assigned to $55 \mathrm{OTU}$, after the removal of low-quality and chimeric sequences. For both $\alpha$ - and $\beta$-diversity analyses, no significant differences were detected between control and LB groups. The same result was also observed when cheese samples were analyzed based on hole dimension (small or large; Figure 2).

With regard to the taxa assigned to each GP sample (Table 3), it must first be noticed that clostridial sequences were not detected in any of the samples. We need to consider that GP cheese contains about 1.5\% $\mathrm{NaCl}$, which constitutes an unfavorable characteristic for clostridial proliferation (IDF, 2017); moreover, the antibacterial enzyme lysozyme is routinely used to contrast the development of these bacteria. Notwithstanding, these measures are frequently insufficient to completely eliminate the problem, particularly in wheels of large dimensions, as in the case of GP, because sometimes the salt, which is provided by immersion of the cheese wheel in concentrated a saline solution, diffuses inside the wheels too slowly to promptly block clostridial growth. We must also consider that, even though the procedure adopted in this study to lyse bacterial cells for DNA extraction was not developed to break the thick endospore structure, clostridial spores were excluded as the causative agent of the defects observed. This is because spores should have germinated and vegetative cells multiplied at the beginning of cheese ripening; therefore this bacterial group should nonetheless have been detected in that form, if present.

Table 2. Primer pairs for 11 obligate heterofermentative lactobacilli, Leuconostoc spp., and Lactobacillus groups used in the present study

\begin{tabular}{|c|c|c|}
\hline Primer $\left(5^{\prime} \rightarrow 3^{\prime}\right)$ & Species & Reference \\
\hline LsC_R_ATGGGGTTCTTGTTGTTATACGG & sanfranciscensis & Zapparoli and Torriani, 1997 \\
\hline bre_IS_F_ATTTTGTTTGAAAGGTGGCTTCGG & Levilactobacillus brevis & Matsuda et al., 2009 \\
\hline buc_IS_R_GGACCAATGGAGCAACTGAA & Lentilactobacillus & Štšepetova et al., 2011 \\
\hline buc_IS_F_AGATTACTGACGCATTGGTTACCA & buchneri & \\
\hline reut_IS_F_ACCGAGAACACCGCGTTATTT & Limosilactobacillus & Štšepetova et al., 2011 \\
\hline ferm_IS_F_AACCGAGAACACCGCGTTAT & fermentum & \\
\hline Leucgrp_F_GCGGCTGCGGCGTCACCTAG & Leuconostoc spp. & Štšepetova et al., 2011 \\
\hline \multicolumn{3}{|l|}{ Leucgrp_R_GGNTACCTTGTTACGACTTC } \\
\hline Lbpb_F_TGGAATTAACGGCTTGCTCTTAC & Lentilactobacillus & Berthoud et al., 2017 \\
\hline Lbpb_R_CAACCCAATTAGGCCAAGGA & parabuchneri & \\
\hline Lbhilgardii_F_AAGCCTGCGAAGGCAAG & Lentilactobacillus & Lee et al., 2010 \\
\hline Lbhilgardii_R_AGGCCACCGGCTTTG & hilgardii & \\
\hline 256_R_GTTACAATGCCGCTGACGAC & wasatchensis & \\
\hline LK1_F_CAACAATCAAAGGGGTTGTTG & Lentilactobacillus kefiri & Toscano et al., 2017 \\
\hline \multicolumn{3}{|l|}{ LK2_R_TCACTAGGAGTAATTGAACCA } \\
\hline Ldel-7_ACAGATGGATGGAGAGCAGA & Lactobacillus spp. & Song et al., 2000 \\
\hline \multicolumn{3}{|l|}{ Lac-2_CCTCTTCGCTCGCCGCTACT } \\
\hline \multicolumn{3}{|l|}{ LU-1'_ATTGTAGAGCGACCGAGAAG } \\
\hline \multicolumn{3}{|l|}{ LU-5_CTAGCGGGTGCGACTTTGTT } \\
\hline LU-3'_AAACCGAGAACACCGCGTT & & \\
\hline
\end{tabular}


Although some studies report the presence of clostridia by $16 \mathrm{~S}$ rRNA analysis of defective and non-defective cheeses (Bassi et al., 2015; Levante et al., 2017; Zago et al., 2021), the results of our analysis clearly indicate that the holes in the examined cheese wheels were not produced by bacteria belonging to this genus.

Sequences attributable to the genus Lactobacillus are extremely abundant, as expected for this type of hard cheese (Neviani et al., 2013; Alessandria et al., 2016), accounting for $96.87 \%$ of the total sequences obtained. Considering the species attribution within the genus, the most represented species by far is Lactobacillus delbrueckii $(76.39 \%)$. This could appear quite uncommon, according to data from conventional microbiological analyses based on colony counts on plates, which detected Lactobacillus helveticus as the most abundant species for this type of cheese (Rossetti et al., 2008). Indeed, our results are in accordance with a recent report by Zago et al. (2021) in a DNA metabarcoding survey on 118 samples of GP cheese obtained from different Italian regions, in which $L$. delbrueckii was found to be one of the most abundant taxa. On the contrary, the presence of $L$. helveticus was dramatically scarce in our samples, as in all controls it never exceeded $1 \%$ of total sequences collected, varying from $0.17 \%$ to $0.91 \%$ of total abundance. Lactobacillus helveticus was also detected at low levels in a study by Zago et al. (2021), in which several samples did not evidence any detectable presence of this species, and only few samples, mainly those coming from Veneto and Trentino-Alto Adige regions, contained more than $10 \%$ L. helveticus in the total population. This apparent contrast with con-
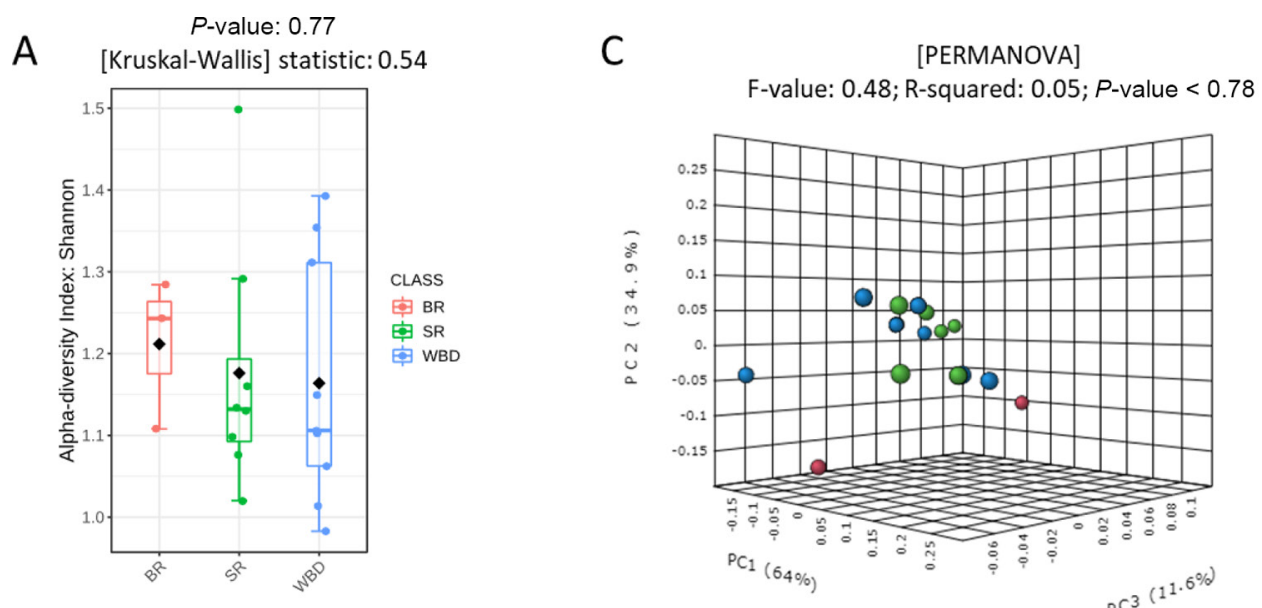

F-value: $0.48 ;$ R-squared: $0.05 ; P$-value $<0.78$

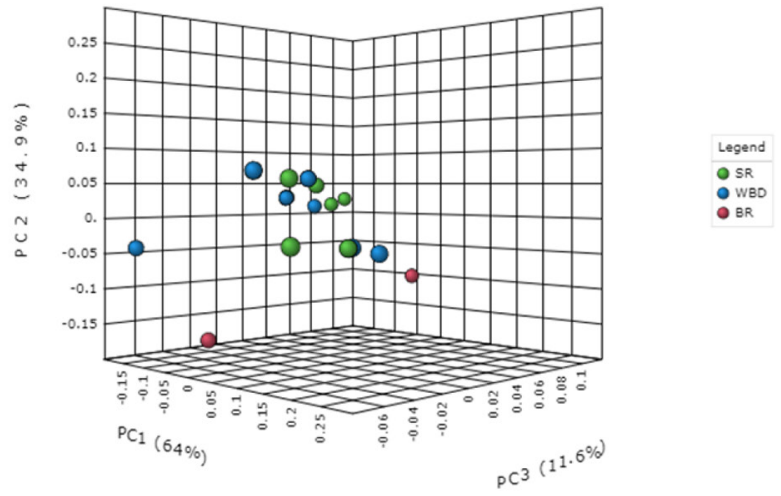

$P$-value: 0.70
B $\quad[$ Kruskal-Wallis] statistic: 0.72
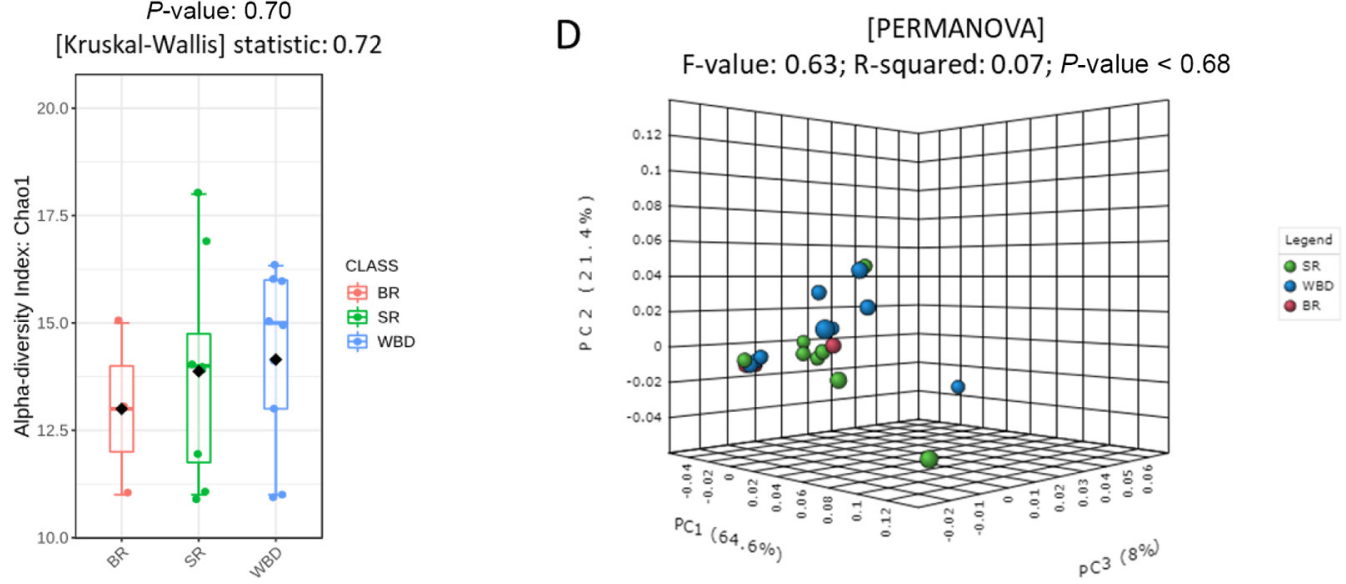

Figure 2. Diversity analyses of cheese samples with large holes (BR group), small holes (SR group), and without blowing defect (WBD group). Panels A and B: $\alpha$-diversity; panels $\mathrm{C}$ and $\mathrm{D}$ : $\beta$-diversity. For panels A and B, horizontal bold lines show the median values. The bottom and top of the boxes show the 25 th and the 75 th percentiles, respectively. The whiskers extend up to the most extreme points within 1.5 times the interquartile ranges. Level of significance: $P \leq 005$. Principal coordinate (PC) analysis based on unweighted (C) and weighted (D) UniFrac distances for the BR, SR, and WBD groups. 


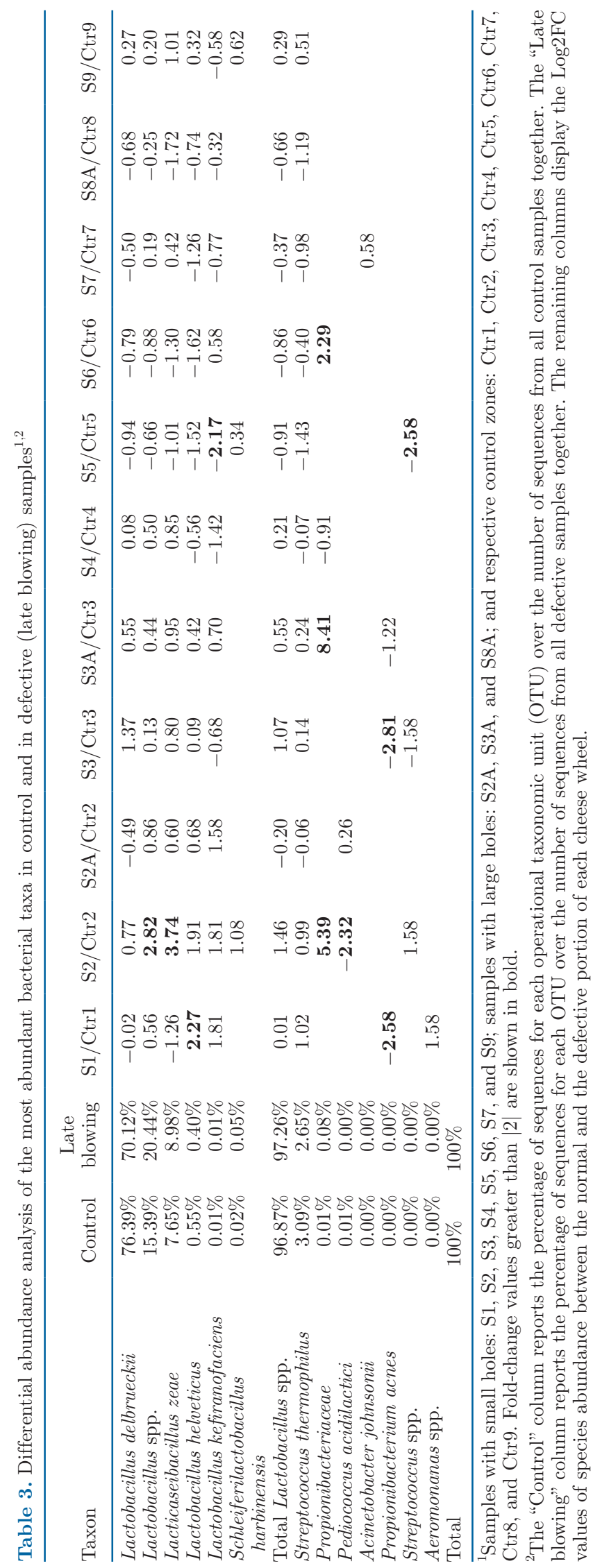

ventional microbiological analyses could be explained by the metabolic status of lactobacilli other than $L$. helveticus, which could have reached higher cell numbers with respect to $L$. helveticus but probably died off during the first months of ripening or entered a nonviable or non-culturable state after long cheese ripening; hence, they were detected by DNA analysis but not with viability tests.

We found around $9.0 \%$ of sequences attributed to Lacticaseibacillus zeae, a species very close to $L$. casei. Its presence was from about $3.5 \%$ up to $20 \%$ of total abundance in our control samples. If we examine the presence of $L$. casei in the study of Zago et al. (2021), we can observe that this species is almost always present, not infrequently in large amounts, up to $50 \%$ or more of the total abundance.

A few Lacticaseibacillus rhamnosus sequences, another member of the L. casei group, were found in only 2 cheese samples (less than $0.01 \%$ ). This is in contrast with what was observed by Zago et al. (2021), where this species was almost always present in GP samples and, in some cases, achieved an abundance around $90 \%$. Considering all lactobacilli, in our control samples we also detected limited numbers of Lactobacillus kefiranofaciens (from $0.008 \%$ up to $0.025 \%$ ), and in 4 samples we found Schleiferilactobacillus harbinensis at a level close to $0.02 \%$ in control samples and $0.05 \%$ in defective samples, which is in accord with the study conducted by Zago et al. (2021). Finally, based on 16S rRNA analysis, $15 \%$ of the Lactobacillus sequences detected were not identified at species level in control samples, whereas this number reached $20 \%$ in GP samples with defect.

Streptococcus thermophilus is known to be frequently present in GP cheese (Gatti et al., 2014; Zago et al., 2021), although at lower levels with respect to lactobacilli. In our study, we detected an average value of $3.1 \%$ relative abundance in the control samples, with values ranging from 1.7 to $3.9 \%$. Zago et al. (2021) found a highly variable situation among the samples analyzed, generally showing low numbers, with only a few cases greater than $10 \%$. Finally, considering the remaining and low-abundant taxa present (at least $0.01 \%$ based on the mean obtained from all control samples), 2 out of 9 samples contained Propionibacterium and Pediococcus acidilactici sequences. We then compared each defective sample with its respective control - that is, cheese from the same wheel in a zone without holes (Table 3). It appears evident that, regarding lactobacilli, no substantial differences were detected that would justify a marked quantitative change in the lactobacilli composition in the defective zones.

The same applies to Strep. thermophilus, indicating no differences in any of the defective samples compared 

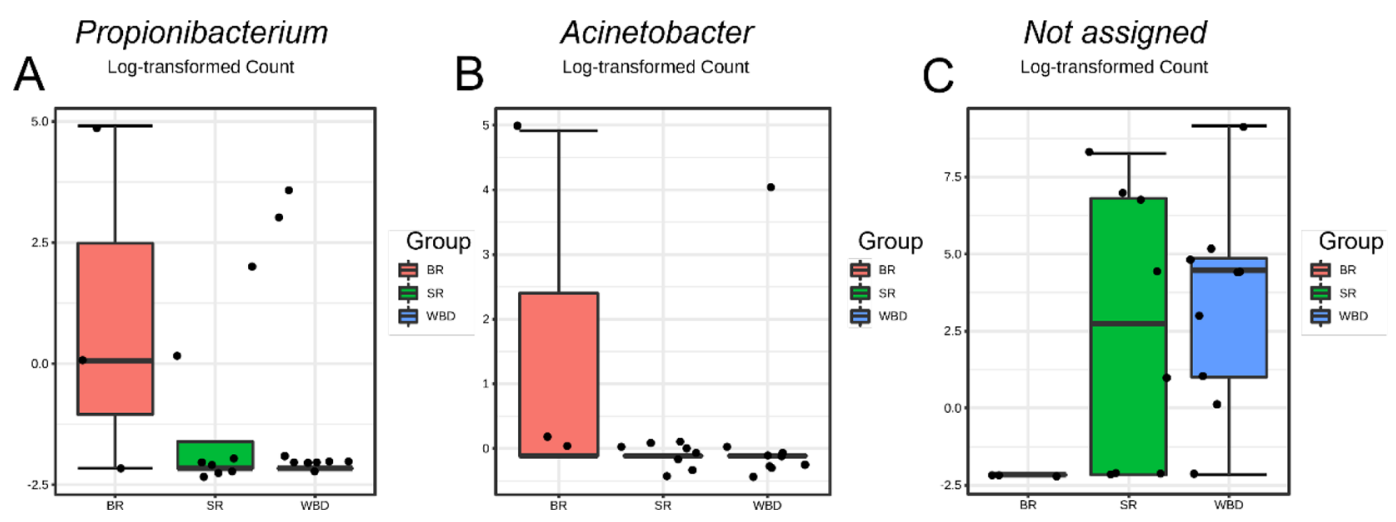

Figure 3. Box plots showing the significant differences in abundance for the genera Acinetobacter (B), Propionibacterium (C), and unassigned sequences (D) after EdgeR analysis. Groups: $\mathrm{BR}=$ big regular holes; $\mathrm{SR}=$ small regular holes; WBD = without blowing defect (control group). Horizontal bold lines show the median values. The bottom and top of the boxes show the 25th and the 75 th percentiles, respectively. The whiskers extend up to the most extreme points within 1.5 times the interquartile ranges.

with the control portions of cheese. This is not surprising, as this species is homofermentative, and therefore it cannot be considered directly responsible for gas production.

As regards the family Propionibacteriaceae, samples $\mathrm{S} 2$, S3A, and S6 evidenced a higher level of these microorganisms in the defective zones compared with their corresponding controls $(\mathrm{S} 2 / \mathrm{Ctr} 2, \log 2 \mathrm{FC}=5.39$; $\mathrm{S} 3 \mathrm{a} / \mathrm{Ctr} 3, \log 2 \mathrm{FC}=8.41 ; \mathrm{S} 6 / \mathrm{Ctr} 6, \log 2 \mathrm{FC}=2.29$ ). Indeed, as reported in Figure $3 \mathrm{~B}$, it is possible to verify via EdgeR that the genus Propionibacterium is more abundant in those samples categorized with big and regular holes $(\log 2 \mathrm{FC}=2.7)$ and, to a lesser extent, in samples with small and regular holes. Because these bacteria (e.g., Propionibacterium freudenreichii ssp. shermanii) are known to be able to develop gas inside cheese wheels (Van Wyk et al., 2018), a situation that can be either of technological importance for some cheeses (e.g., Emmental) or, in contrast, associated with the presence of LB defects (McSweeney, 2007), we could hypothesize that these defects may be caused by such a bacterial category.

Although the remaining categories identified have low relative abundance values, and it is therefore unlikely that these groups make any significant contribution to defect formation, sequences assigned to members of the genus Acinetobacter are highly present in samples with big and regular holes (Figure $3 \mathrm{C}, \log 2 \mathrm{FC}=3.3$ ). As described elsewhere (Doulgeraki et al., 2012; Zago et al., 2021), this genus has raw milk as a source and, although not associated with the origin of LB defects, is considered an undesirable food spoilage member of resident bacteria in GP processing plants. Last, EdgeR analysis revealed that sequences without a taxonomical assignment are more abundant in cheese samples collected from small holes (Figure 3D). After using MegaBLAST to verify the nucleotide sequences of those OTU classified as not available, including de novo OTU, it is possible to verify that these sequences originate mainly from members of the genus Lactobacillus, such as L. delbrueckii ssp. delbrueckii NBRC 3202 (accession number: AP019750.1; identity: 100\%; E-value: 6e-131), L. rhamnosus strain LV108 (accession number: CP053619.1; identity: 98.7\%; E-value: 1e-122), S. harbinensis strain LH991 (accession number: CP045180.1; identity: 99.61\%; E-value: 3e-129), and L. casei strain FBL6 (accession number: CP074377.1; identity: 100\%; E-value: 6e-131).

Because, according to the genetic analysis, it was not possible to identify a specific bacterial group clearly linkable to the presence of the defect in cheese samples with holes classified as "small and regular," we decided to better inspect a possible involvement of heterofermentative lactobacilli. The genus Lactobacillus includes obligate homofermentative lactobacilli, facultative heterofermentative lactobacilli, and obligate heterofermentative lactobacilli (OHL) species (Pot et al., 2014). Only this last group comprises microbes able to produce $\mathrm{CO}_{2}$ when fermenting hexoses. Among the Lactobacillus species detected by metataxonomic analysis, 3 were obligate homofermentative ( $L$. delbrueckii, L. helveticus, and L. kefiranofaciens) and 3 were facultative heterofermentative lactobacilli (L. zeae, S. harbinensis, and L. rhamnosus), whereas no OHL species were detected, although it must be considered that $15 \%$ of the Lactobacillus sequences were not identified at the species level. The only sugar initially present in milk is the disaccharide lactose, but during cheese ripening other sugars can became available, such as galactose from lactose hydrolysis, exported from bacteria unable to use 

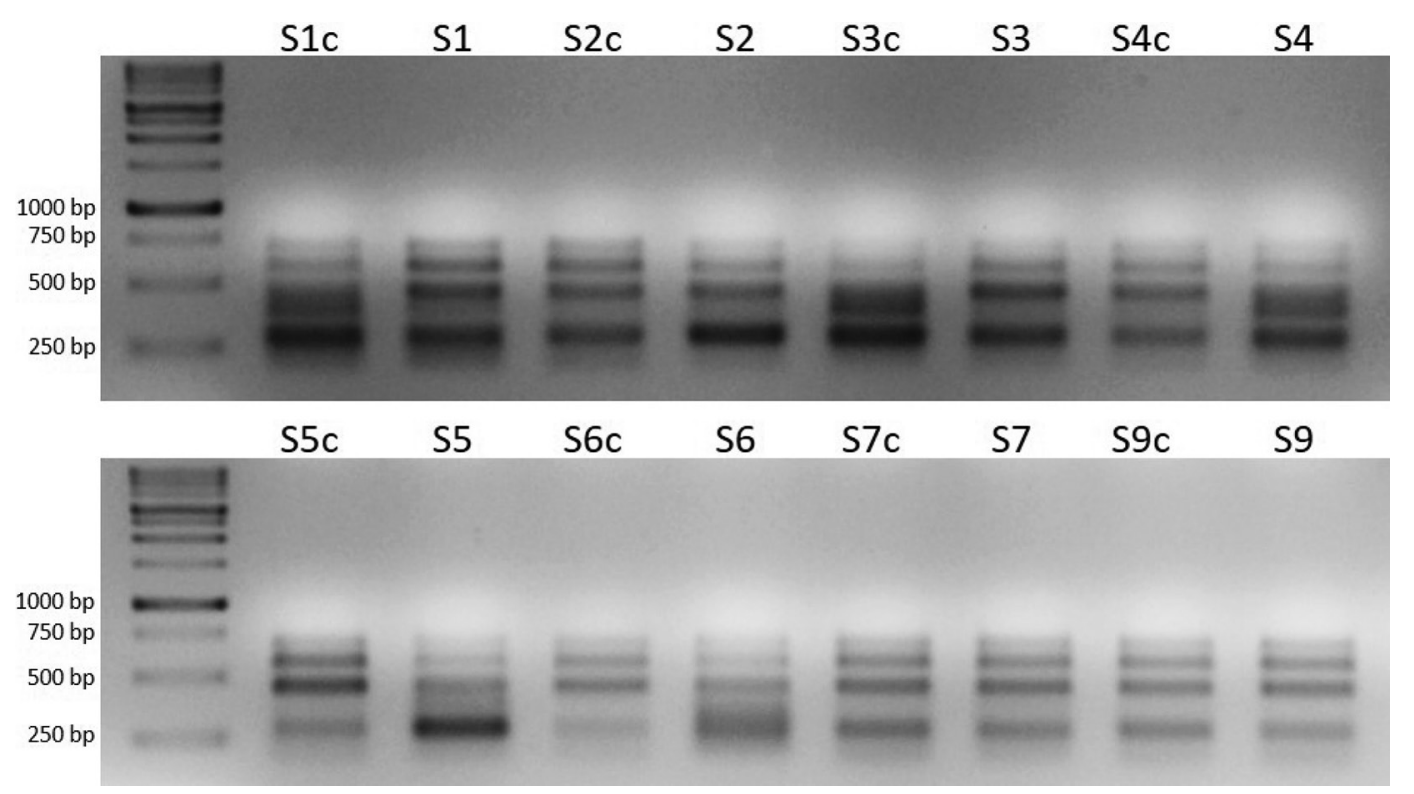

Figure 4. Agarose gel electrophoresis of PCR products from multiplex PCR differentiating lactobacilli into 4 groups: Group I, obligate homofermentative, $\sim 450$ bp: Lactobacillus delbrueckii ssp. bulgaricus and L. delbrueckii ssp. lactis; Group II, obligate homofermentative, $\sim 300$ bp: Lactobacillus acidophilus, Lactobacillus amylovorus, Lactobacillus crispatus, Lactobacillus helveticus, Lactobacillus gasseri, and Lactobacillus jensenii; Group III, facultative and obligate heterofermentative, $\sim 400$ bp: Lacticaseibacillus paracasei ssp. paracasei, L. paracasei ssp. tolerans, Lacticaseibacillus casei, and Lacticaseibacillus rhamnosus; Group IV, facultative heterofermentative, obligate heterofermentative, and obligate homofermentative, $\sim 350$ bp: Ligilactobacillus salivarius ssp. salivarius, L. salivarius ssp. salicinius, Limosilactobacillus reuteri, Limosilactobacillus fermentum, and Lactiplantibacillus plantarum. Lane 1 =1-kb DNA ladder. S1 to S9 are samples; and S1c to S9c represent their corresponding controls.

it, and other monosaccharides, such as ribose, coming from bacterial cells lysis during prolonged cheese ripening (Ortakci et al., 2015), which can be fermented by OHL with production of gas.

For this reason, we decided to perform a specific genetic test by DNA amplification to look for the possible presence of the most widespread OHL, namely Fructilactobacillus sanfranciscensis, Levilactobacillus brevis, Lentilactobacillus buchneri, Limosilactobacillus reuteri, Limosilactobacillus fermentum, Lentilactobacillus parabuchneri, Lentilactobacillus hilgardii, Fructilactobacillus fructivorans, Fructilactobacillus lindneri, Paucilactobacillus wasatchensis, and Lentilactobacillus kefiri by using species-specific primer couples. This analysis did not detect the presence of any of the OHL species mentioned previously in any of the samples, which is in accordance with the metataxonomic analysis.

As a further approach, we also used 3 broader primer couples, based on the $16 \mathrm{~S}$ to $23 \mathrm{~S}$ rRNA intergenic spacer regions (Song et al., 2000) in a multiplex amplification experiment. The results of this analysis are reported in Figure 4. By looking at each defective sample compared with its respective control, some qualitative differences were visible from the different fingerprints. Samples 5 and 6 show a markedly different fingerprint from their respective controls; samples 7 and 9 appear identical to their controls; and samples 1,2, 3, and 4 evidence different intensities of some bands with respect to their controls. Overall, this result reveals that in most of the samples (6 out of 8 ) an unbalanced distribution of Lactobacillus groups is observed; the consequence of this on the development of LB defect deserves further investigations.

\section{CONCLUSIONS}

Based on the results obtained in the present study, we can affirm that the presence of holes in most of the cheese samples examined is not determined by a clearly identifiable bacterial species, as detected by $16 \mathrm{~S}$ rRNA analysis. Our DNA analysis of OHL did not evidence a significant presence of any of the most widespread obligate homofermentative species. On the contrary, a broader fingerprinting analysis based on intergenic spacer regions amplification revealed some differences in the composition of the Lactobacillus population in the area surrounding the defects, with respect to the non-defective zones of the same cheese wheel. Further analyses are therefore needed to better study the microbiota and identify these deleterious bacteria and their involvement in the process of eye formation. 


\section{ACKNOWLEDGMENTS}

This work was funded by Regione Veneto, Venezia, Italy, under the "Nuovo piano industriale del lattierocaseario Veneto" (NIP) project (POR-FESR 20142020). The authors have not stated any conflicts of interest.

\section{REFERENCES}

Alessandria, V., I. Ferrocino, F. De Filippis, M. Fontana, K. Rantsiou, D. Ercolini, and L. Cocolin. 2016. Microbiota of an Italian Granalike cheese during manufacture and ripening, unraveled by $16 \mathrm{~S}$ rRNA-based approaches. Appl. Environ. Microbiol. 82:3988-3995. https://doi.org/10.1128/AEM.00999-16.

Bassi, D., E. Puglisi, and P. S. Cocconcelli. 2015. Understanding the bacterial communities of hard cheese with blowing defect. Food Microbiol. 52:106-118. https://doi.org/10.1016/j.fm.2015.07.004.

Bava, L., J. Bacenetti, G. Gislon, L. Pellegrino, P. D'Incecco, A. Sandrucci, A. Tamburini, M. Fiala, and M. Zucali. 2018. Impact assessment of traditional food manufacturing: The case of Grana Padano cheese. Sci. Total Environ. 626:1200-1209. https://doi .org/10.1016/j.scitotenv.2018.01.143.

Bermúdez, J., M. J. González, J. A. Olivera, J. A. Burgueño, P. Juliano, E. M. Fox, and S. M. Reginensi. 2016. Seasonal occurrence and molecular diversity of clostridia species spores along cheesemaking streams of 5 commercial dairy plants. J. Dairy Sci. 99:3358-3366. https://doi.org/10.3168/jds.2015-10079.

Berthoud, H., D. Wüthrich, R. Bruggmann, D. Wechsler, M.-T. Fröhlich-Wyder, and S. Irmler. 2017. Development of new methods for the quantitative detection and typing of Lactobacillus parabuchneri in dairy products. Int. Dairy J. 70:65-71. https://doi.org/ 10.1016/j.idairyj.2016.10.005.

Bowen, I. 2018. Growth characteristics of Lactobacillus wasatchensis and its detection and enumeration using quantitative polymerase chain reaction. MS Thesis. Department of Nutrition, Dietetics, and Food Sciences, Utah State University, Logan.

D'Incecco, P., L. Pellegrino, J. A. Hogenboom, P. S. Cocconcelli, and D. Bassi. 2018. The late blowing defect of hard cheeses: Behaviour of cells and spores of Clostridium tyrobutyricum throughout the cheese manufacturing and ripening. Lebensm. Wiss. Technol. 87:134-141. https://doi.org/10.1016/j.lwt.2017.08.083.

De Filippis, F., A. La Storia, G. Stellato, M. Gatti, and D. Ercolini. 2014. A selected core microbiome drives the early stages of three popular Italian cheese manufactures. PLOS One 9:e89680. https:/ /doi.org/10.1371/journal.pone.0089680.

Dhariwal, A., J. Chong, S. Habib, I. L. King, L. B. Agellon, and J. Xia. 2017. MicrobiomeAnalyst: A web-based tool for comprehensive statistical, visual and meta-analysis of microbiome data. Nucleic Acids Res. 45(W1):W180-W188. https://doi.org/10.1093/ nar/gkx295.

Doulgeraki, A. I., D. Ercolini, F. Villani, and G.-J. E. Nychas. 2012. Spoilage microbiota associated to the storage of raw meat in different conditions. Int. J. Food Microbiol. 157:130-141. https://doi .org/10.1016/j.ijfoodmicro.2012.05.020.

Gatti, M., B. Bottari, C. Lazzi, E. Neviani, and G. Mucchetti. 2014. Invited review: Microbial evolution in raw-milk, long-ripened cheeses produced using undefined natural whey starters. J. Dairy Sci. 97:573-591. https://doi.org/10.3168/jds.2013-7187.

IDF (International Dairy Federation). 2017. The importance of salt in the manufacturing and ripening of cheese. IDF Factsheet 001/2017-13. International Dairy Federation.

Lee, M.-K., K.-H. Ku, Y.-J. Kim, K.-H. Kim, Y.-R. Kim, and H.J. Yang. 2010. Development of species-specific primers for PCR identification of Lactobacillus hilgardii and Lactobacillus farciminis in kimchi. Prev. Nutr. Food Sci. 15:159-166. https://doi.org/10 $.3746 /$ jfn.2010.15.2.159.
Levante, A., F. De Filippis, A. La Storia, M. Gatti, E. Neviani, D. Ercolini, and C. Lazzi. 2017. Metabolic gene-targeted monitoring of non-starter lactic acid bacteria during cheese ripening. Int. J. Food Microbiol. 257:276-284. https://doi.org/10.1016/j.ijfoodmicro .2017.07.002.

Matsuda, K., H. Tsuji, T. Asahara, K. Matsumoto, T. Takada, and K. Nomoto. 2009. Establishment of an analytical system for the human fecal microbiota, based on reverse transcription-quantitative PCR targeting of multicopy rRNA molecules. Appl. Environ. Microbiol. 75:1961-1969. https://doi.org/10.1128/AEM.01843-08.

Mayo, B., J. Rodríguez, L. Vázquez, and A. B. Flórez. 2021. Microbial interactions within the cheese ecosystem and their application to improve quality and safety. Foods 10:602. https://doi.org/10 $.3390 /$ foods 10030602 .

McSweeney, P. L. H.. 2007. Grana-type cheeses and Parmesan. Pages 208-213 in Cheese Problems Solved. P. L. H. McSweeney, ed. Elsevier. https://doi.org/10.1533/9781845693534.208.

Neviani, E., B. Bottari, C. Lazzi, and M. Gatti. 2013. New developments in the study of the microbiota of raw-milk, long-ripened cheeses by molecular methods: The case of Grana Padano and Parmigiano Reggiano. Front. Microbiol. 4. https://doi.org/10.3389/ fmicb.2013.00036.

Ortakci, F., J. R. Broadbent, C. J. Oberg, and D. J. McMahon. 2015. Late blowing of Cheddar cheese induced by accelerated ripening and ribose and galactose supplementation in presence of a novel obligatory heterofermentative nonstarter Lactobacillus wasatchensis. J. Dairy Sci. 98:7460-7472. https://doi.org/10.3168/jds.2015 -9468 .

Picozzi, C., F. D'Anchise, and R. Foschino. 2006. PCR detection of Lactobacillus sanfranciscensis in sourdough and Panettone baked product. Eur. Food Res. Technol. 222:330-335. https://doi.org/10 .1007/s00217-005-0121-z.

Pot, B., G. E. Felis, K. De Bruyne, E. Tsakalidou, K. Papadimitriou, J. Leisner, and P. Vandamme. 2014. The genus Lactobacillus. Pages 249-353 in Lactic Acid Bacteria: Biodiversity and Taxonomy. W. P. Holzapfel and B. J. B. Wood, ed. John Wiley \& Sons Inc.

Robinson, M. D., D. J. McCarthy, and G. K. Smyth. 2010. edgeR: A Bioconductor package for differential expression analysis of digital gene expression data. Bioinformatics. 26:139-140. https://doi.org/ 10.1093/bioinformatics/btp616.

Rossetti, L., M. E. Fornasari, M. Gatti, C. Lazzi, E. Neviani, and G. Giraffa. 2008. Grana Padano cheese whey starters: Microbial composition and strain distribution. Int. J. Food Microbiol. 127:168171. https://doi.org/10.1016/j.ijfoodmicro.2008.06.005.

Settanni, L., D. van Sinderen, J. Rossi, and A. Corsetti. 2005. Rapid differentiation and in situ detection of 16 sourdough Lactobacillus species by multiplex PCR. Appl. Environ. Microbiol. 71:30493059. https://doi.org/10.1128/AEM.71.6.3049-3059.2005.

Song, Y., N. Kato, C. Liu, Y. Matsumiya, H. Kato, and K. Watanabe. 2000. Rapid identification of 11 human intestinal Lactobacillus species by multiplex PCR assays using group- and species-specific primers derived from the 16S-23S rRNA intergenic spacer region and its flanking 23S rRNA. FEMS Microbiol. Lett. 187:167-173. https://doi.org/10.1111/j.1574-6968.2000.tb09155.x.

Štšepetova, J., E. Sepp, H. Kolk, K. Lõivukene, E. Songisepp, and M. Mikelsaar. 2011. Diversity and metabolic impact of intestinal Lactobacillus species in healthy adults and the elderly. Br. J. Nutr. 105:1235-1244. https://doi.org/10.1017/S0007114510004770.

Toscano, M., R. De Grandi, V. L. Miniello, R. Mattina, and L. Drago. 2017. Ability of Lactobacillus kefiri LKF01 (DSM32079) to colonize the intestinal environment and modify the gut microbiota composition of healthy individuals. Dig. Liver Dis. 49:261-267. https://doi.org/10.1016/j.dld.2016.11.011.

Treu, L., P. G. Kougias, B. de Diego-Díaz, S. Campanaro, I. Bassani, J. Fernández-Rodríguez, and I. Angelidaki. 2018. Two-year microbial adaptation during hydrogen-mediated biogas upgrading process in a serial reactor configuration. Bioresour. Technol. 264:140-147. https://doi.org/10.1016/j.biortech.2018.05.070.

Van Wyk, J., R. A. Morkel, and L. Dolley. 2018. Metabolites of Propionibacterium: Techno- and biofunctional ingredients. Pages 
205-260 in Handbook of Food Bioengineering, Alternative and Replacement Foods. A. M. Holban and A. M. Grumezescu, ed. Academic Press. https://doi.org/10.1016/B978-0-12-811446-9.00008-3

Yasui, T., H. Taguchi, and T. Okamoto. 1997. A specific oligonucleotide primer for the rapid detection of Lactobacillus lindneri by polymerase chain reaction. Can. J. Microbiol. 43:157-163. https:/ /doi.org/10.1139/m97-021.

Zago, M., T. Bardelli, L. Rossetti, N. Nazzicari, D. Carminati, A. Galli, and G. Giraffa. 2021. Evaluation of bacterial communities of Grana Padano cheese by DNA metabarcoding and DNA fingerprinting analysis. Food Microbiol. 93:103613. https://doi.org/10 $.1016 / j . f m .2020 .103613$.

Zapparoli, G., and S. Torriani. 1997. Rapid identification and detection of Lactobacillus sanfrancisco in sourdough by species-specific PCR with 16S rRNA-targeted primers. Syst. Appl. Microbiol. 20:640-644. https://doi.org/10.1016/S0723-2020(97)80036-7.
Zheng, J., S. Wittouck, E. Salvetti, C. M. A. P. Franz, H. M. B. Harris, P. Mattarelli, P. W. O'Toole, B. Pot, P. Vandamme, J. Walter, K. Watanabe, S. Wuyts, G. E. Felis, M. G. Gänzle, and S. Lebeer. 2020. A taxonomic note on the genus Lactobacillus: Description of 23 novel genera, emended description of the genus Lactobacillus Beijerinck 1901, and union of Lactobacillaceae and Leuconostocaceae. Int. J. Syst. Evol. Microbiol. 70:2782-2858. https://doi.org/ 10.1099/ijsem.0.004107.

\section{ORCIDS}

Vinícius da Silva Duarte (ㄱ https://orcid.org/0000-0002-0356-3947

Alessio Giacomini $\odot$ https://orcid.org/0000-0003-4124-0142 